\title{
Influence of nitrogen on CO-laser characteristics
}

\author{
Galina M. Grigorian ${ }^{1}$, Adam Cenian ${ }^{* 2}$ \\ ${ }^{1}$ Faculty of Physics, St. Petersburg State University, 199034 St. Petersburg, \\ ${ }^{2}$ The Szewalski Institute of Fluid-Flow Machinery, Polish Academy of Sciences, Fiszera 14, 80-952 Gdansk.
}

Received October 03, 2016; accepted March 13, 2017; published June 30, 2016

\begin{abstract}
The effect of a nitrogen addition on plasma discharge of $\mathrm{CO}$ lasers in plasma-chemical processes is discussed here. It is shown that a nitrogen addition improves the laser characteristics and changes the composition of a laser active medium. The reduction of CO highlyexcited vibrational-states populations with a current is smaller in the case of mixtures with nitrogen additions. The addition of nitrogen significantly decreases the $\mathrm{CO}$ dissociation level and concentration of $\mathrm{C}$ atoms created in plasma-chemical reactions of a laser discharge.
\end{abstract}

The processes of molecular dissociation, following plasma-chemistry, changing the composition of a laser active-medium and laser characteristics, determine the long-term effective operation of molecular sealed-off lasers (working without a gas exchange in the laser discharge tube).

These processes are extremely important for the CO-laser operation. The vibrationally excited $\operatorname{CO}\left(\mathrm{X}^{1} \Sigma\right.$, v) molecules, involved in laser-radiation generation, hardly relax during collisions with the initial component of an active mixture, however the ratio of their relaxation could significantly increase when the collision partners are some products of the above mentioned plasma-chemical processes [1]. It is well known (see e.g. [2] and the references therein), that in the sealed-off CO-lasers usually mixtures with significant concentrations of nitrogen are applied, which supports better energycharacteristics and improves the long-life performance of the laser.

The addition of nitrogen molecules to the laser activemixture results in an increase of population of vibrationally excited $\mathrm{CO}$ molecules due to vibrationalvibrational $\left(\mathrm{VV}^{\prime}\right)$ relaxation processes. However, there are some important indicators pointing to the fact that improvements of CO-laser operation after a nitrogen addition does not necessary result from the additional mechanism of $\mathrm{CO}$ states excitation. The addition of nitrogen to an He-CO mixture leads to a significant change in plasma-chemical products. Firstly, some new nitrogen-containing products appear: $\mathrm{N}$ atoms, molecules $\mathrm{CN}$, NO, etc. Secondly, nitrogen and nitrogen-containing products influence the plasma composition of an initial He-CO mixture.

*E-mail: cenian@imp.gda.pl
Up to now the influence of nitrogen on plasmachemistry of a $\mathrm{CO}$ laser has rarely been investigated. There is a lack of comprehensive models for plasmachemistry of He-CO- $\mathrm{N}_{2}$ mixtures (except some common feature with the plasma of a $\mathrm{CO}_{2}$ laser [3-5]), which would help to estimate the level of degradation of the initial laser mixture. However, it has been shown in [5] that the $\mathrm{CO}_{2}$ laser plasma with a high nitrogen content, $30 \%<\left[\mathrm{N}_{2}\right]<$ $50 \%$, ensuring proper storage of vibrational energy in $\mathrm{CO}_{2}$ lasers, is characterized by low electron temperature and thus, small chemical reactivity, i.e. a small level of $\mathrm{CO}_{2}$ dissociation. From the investigations it follows that mixture decomposition decreases with an increase of $\mathrm{N}_{2}$ content in $\mathrm{CO}_{2}$ laser mixtures. In contrast, electron temperature does not decrease after nitrogen additions to He-CO plasma. The temperature is constant or even slightly increases (up to $15 \%$ ) after nitrogen additions.

However, there is a small amount of experimental data on products of plasma-chemistry in such discharges and on products dependence on experimental conditions and discharge time-duration. The concentrations of $\mathrm{CN}$ molecules have been measured in flowing $\mathrm{He}-\mathrm{CO}-\mathrm{N}_{2}$ discharges [6-8] and in sealed-off systems, and theoretically determined based on a model of plasmachemical processes - see Ref. [9].

Thus, the aim of this work is to determine the influence of nitrogen additions to $\mathrm{He}-\mathrm{CO}$ mixtures on $\mathrm{CO}$-laser characteristics, including $\mathrm{CO}$ vibrational excitation. The experiments were performed under conditions typical for discharge tubes of CO-laser working in sealed-off and gas flowing regimes.

The experimental set-up has been already described in a previous work [9]. The water-cooled discharge tube from molybdenum glass, $17 \mathrm{~mm}$ in diameter, was used in the experiment. The lengths of a discharge zone was $50 \mathrm{~cm}$. Hollow cylindrical electrodes from tantalum were installed in the side branches of the discharge tube at a distance of $4 \mathrm{~cm}$ from its axis. The discharge was excited using DC currents of 10-100 mA. The experiment was performed for initial $\mathrm{He}-\mathrm{CO}$ and $\mathrm{He}-\mathrm{CO}-\mathrm{N}_{2}$ mixtures. Relative $\mathrm{CO}$ and $\mathrm{N}_{2}$ concentrations were varied in the range $3 \div 15 \%$. The gas pressure was set at $5 \div 15$ Torr. The setup can work in sealed-off and gas-flowing regimes (gas velocities $1 \div 3 \mathrm{~m} / \mathrm{s}$ were applied). Initially, the gas 
mixtures used in the experiments were carefully purified by using a system of traps with silica-gel and zeolite.

The emission spectra from the discharge positivecolumn were registered using a spectrometer in the range $300 \div 6000 \mathrm{~nm}$, in order to determine the respective populations of vibrationally excited states of $\mathrm{CO}\left(\mathrm{X}^{1} \Sigma\right)$ and gas temperature along the symmetry axis of the discharge tube as well as its radial profile. The determination was done by analysing the rotational structure of $\mathrm{CO}$ vibrational bands.

The vibrational distribution of $\mathrm{CO}$ molecules in the ground electronic state $\left(\mathrm{X}^{1} \Sigma\right)$ was determined from the spectra of IR (infrared) molecular emission at the $(\mathrm{v} \rightarrow \mathrm{v}-1)$ fundamental frequency, and the first $(\mathrm{v} \rightarrow \mathrm{v}-2)$ overtone.

During the experiment the overtones emission was registered in the neighbourhood of the symmetry axis $(\sim 3$ $\mathrm{mm}$ ) in a discharge positive column. The spectra were studied using a Czerny-Turner monochromator with a diffraction grating 300 and 1200 lines $/ \mathrm{mm}$. Cooled (by liquid nitrogen) photoresistors $\mathrm{HgCdTl}$ and uncooled $\mathrm{InSb}$ were used as photodetectors.

The lack of cataphoresis significant influence during the measurements in systems without gas flows was proved. It was confirmed by registering $\mathrm{He}$ line intensities and $\mathrm{CO}$ molecular band at various points along the symmetry axis of the discharge tube using an optical waveguide.

The electric field in the discharge was determined by measuring the voltage drops across the discharge gap and the electrode sheaths. The electrode sheath voltages were found from the voltage measurements in experiments with different discharge lengths. A typical electrode sheath voltage was $350 \div 400 \mathrm{~V}$. The estimated value of the reduced electric field $E / N$ was $(2.1 \div 3.2) \cdot 10^{-16} \mathrm{~V} \mathrm{~cm}^{2}$.

Table 1. The $\mathrm{CO}$ vibrational temperature $[\mathrm{K}]$ under different conditions

\begin{tabular}{|l|l|l|l|l|}
\hline \multirow{2}{*}{$\begin{array}{l}\text { Pressure } \\
\text { Current }\end{array}$} & \multicolumn{2}{|c|}{5 Torr } & \multicolumn{2}{c|}{10 Torr } \\
\cline { 2 - 5 } & $50 \mathrm{~mA}$ & $70 \mathrm{~mA}$ & $50 \mathrm{~mA}$ & $70 \mathrm{~mA}$ \\
\hline $\mathrm{He}-\mathrm{CO}=$ & $1750 /$ & 1950 & $1700 /$ & 1800 \\
$95: 5$ & $\mathbf{1 7 0 0}$ & & $\mathbf{1 6 0 0}$ & \\
\hline $\mathrm{He}-\mathrm{CO}=$ & 1700 & 1800 & 1700 & 1800 \\
$91: 9$ & & & & \\
\hline $\mathrm{He}-\mathrm{CO}-\mathrm{N}_{2}=$ & $2850 /$ & 2800 & $2700 /$ & 2750 \\
$95: 3: 2$ & $\mathbf{2 7 0 0}$ & & $\mathbf{2 6 0 0}$ & \\
\hline $\mathrm{He}-\mathrm{CO}-\mathrm{N}_{2}=$ & $2450 /$ & 2500 & $2500 /$ & 2650 \\
$95: 4: 1$ & $\mathbf{2 4 0 0}$ & & $\mathbf{2 4 0 0}$ & \\
\hline
\end{tabular}

The measurements were performed for a CO-lasergeneration regime and without laser-radiation generation. In order to enforce laser generation a resonator in a flat/spherical configuration was applied. A fully reflecting metallic mirror (steel covered with gold) had a $5 \mathrm{~m}$ curvature radius. The exit mirror (with transmission $85 \div 95 \%$ ) was a flat plate of $\mathrm{CaF}_{2}$ or $\mathrm{ZnSe}$ covered by a dielectric multilayer. The laser energy characteristics under the gas-flow regime were: power $0.4 \mathrm{~W}$ and electrooptic efficiency $\eta=10 \%$; under sealed-off regime $0.2 \mathrm{~W}$ and $\eta=4.8 \%$, respectively. The resonator was not optimised for generation conditions.

The measurements showed that a nitrogen addition to the $\mathrm{He}-\mathrm{CO}$ laser medium results in a substantial increase of $\mathrm{CO}$ vibrational temperatures. This should be related to the transfer of vibrational energy from nitrogen to carbon oxide due to the $\mathrm{VV}^{\prime}$ exchange. As a result, a stationary state is realised in the laser medium, with a high level of vibrational energy in $\mathrm{CO}$ molecules.

Table 1 presents the results of vibrational temperature $\left(\mathrm{T}_{1}\right)$ determination:

$$
T_{l}=E_{l} / \ln \left(N_{0} / N_{l}\right)
$$

where $E_{l}$ - the energy of the first excited vibrational state; $\mathrm{N}_{0}$ and $\mathrm{N}_{1}$ - the populations of the ground and first excited vibrational state of $\mathrm{CO}$ molecule. The numbers in Table 1 given in a normal font correspond to the measurements in the gas-flow regime and the numbers in bold - to the sealed-off one. As can be seen, the values for both regimes are relatively close. The reproducibility of results was estimated as $10 \%$.

The presence of nitrogen in the mixture results in increased populations of $\mathrm{CO}$ excited vibrational-states, and this effect grows with the considered nitrogen concentrations. Laser generation was observed in He-CO$\mathrm{N}_{2}$ mixtures (at the level $0.4 \mathrm{~W}$ ) but it wasn't observed when nitrogen was absent in the mixture under any considered experimental conditions.

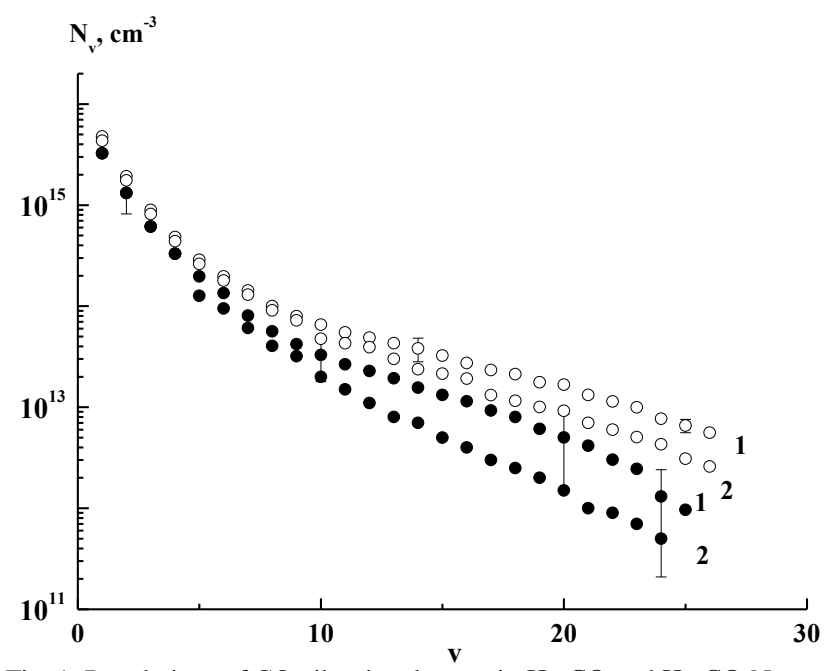

Fig. 1. Populations of $\mathrm{CO}$ vibrational states in $\mathrm{He}-\mathrm{CO}$ and $\mathrm{He}-\mathrm{CO}-\mathrm{N}_{2}$ mixtures without laser-radiation generation at various discharge currents; black full circles - He:CO=91:9 mixture, open circles He:CO: $\mathrm{N}_{2}=91: 4: 5$ mixture; curves $1: \mathrm{I}=25 \mathrm{~mA}, 2: \mathrm{I}=40 \mathrm{~mA}$, pressure $\mathrm{P}=12$ Torr. 
It was found that a decrease of $\mathrm{CO}$ vibrational statespopulations in the mixtures containing nitrogen proceeds under much higher energy inputs (currents) than in the mixtures without nitrogen. Figure 1 presents $\mathrm{CO}$ vibrational energy distributions for mixtures $\mathrm{He}-\mathrm{CO}$ and $\mathrm{He}-\mathrm{CO}-\mathrm{N}_{2}$ for different current values. It can be seen that populations in the $\mathrm{He}-\mathrm{CO}-\mathrm{N}_{2}$ mixture fall with significantly slower current values. It should be pointed out that the gas temperatures measured (near symmetry axis) in both mixtures $\mathrm{He}-\mathrm{CO}$ and $\mathrm{He}-\mathrm{CO}-\mathrm{N}_{2}$ were similar and varied in the range $360-480 \mathrm{~K}$. These temperatures do not depend on gas flow.

One of the main causes of a smaller decrease of $\mathrm{CO}$ vibrational states-populations under increase of a current value in the mixtures with nitrogen is probably related to a lower CO-dissociation level and lower concentrations of plasma-chemical decomposition products. This should be proved in future investigations.

In conclusion, the performed measurements under conditions characteristic for a plasma discharge. In a $\mathrm{CO}$ laser showed that a nitrogen addition to the $\mathrm{He}-\mathrm{CO}$ mixture leads to significant increases in $\mathrm{CO}$ vibrational temperature as well as populations of $\mathrm{CO}$ excited states. The reduction of $\mathrm{CO}$ highly-excited vibrational-states populations with a current is smaller in the case of mixtures with nitrogen additions. It was also found from the IR spectra that the $\mathrm{CO}$ dissociation decreases in the plasma with nitrogen.

More detailed investigations of plasma-chemistry dependence (especially CO concentration) on a nitrogen content should be performed in the future.

This work has been supported by St. Petersburg University (grant No. 11.38.203.2014).

\section{References}

[1] G.M. Grigorian, I.V. Kochetov, Plasma Phys. Rep. 30, 788 (2004).

[2] V.S. Aleinikov, V.I. Masychev, CO Lasers (Moscow, Radio i Svyaz' 1990)

[3] A. Cenian, A. Chernukho, V. Borodin, G. Śliwiński, Contr. Plasma Phys. 34, 25 (1994)

[4] A. Cenian, A. Chernukho, V. Borodin, Contrib. Plasma Phys. 35, 273 (1995).

[5] A. Cenian, A. Chernukho, P. Kukiełło, R. Zaremba, V. Borodin, G. Śliwiński, J.Phys. D: Appl. Phys. 30, 1103 (1997).

[6] E.A. Trubacheev, Trudy FIAN 102, 3 (1977).

[7] G.M. Grigorian, B.M. Dymshits, Yu.Z. Ionikh, Sov. J. Quant. Electron. 19, 889 (1989).

[8] V.N. Ochkin, S. Yu. Savinov, N.N. Sobolev, E.A. Trubacheev, Kvant. Elektr. 1, 573 (1974).

[9] G.M. Grigorian, A. Cenian, Plasma Chem. Plasma Process. 31, 337 (2011) 